\title{
Nonconceptualism, Hume's Problem, AND THE DEDUCTION
}

\author{
Anil Gomes \\ Trinity College, University of Oxford \\ Forthcoming Philosophical Studies [accepted September 2016] \\ For a symposium on Lucy Allais's Manifest Reality: Kant's Idealism and his \\ Realism (Oxford University Press, 2015)
}

\section{Introduction}

Lucy Allais's Manifest Reality: Kant's Idealism and his Realism (Oxford University Press, 2015) sets out and defends a moderate metaphysical interpretation of Kant's transcendental idealism, one which aims to avoid the pitfalls of both immoderate phenomenalist readings and overly modest deflationary readings. The result is a forceful and articulate take on some of the fundamental topics in the first Critique: the nature of intuition; the problem posed by synthetic a priori judgements; the mind-dependence of appearances and their relation to things as they are in themselves; the role of idealism in explaining metaphysical knowledge. I found myself agreeing with many of the overarching themes of her book, and admiring much of her articulation of those themes. My comments here confine myself to one issue: Allais's reading of the Transcendental Deduction of the Categories in Chapters 11 and 12 of her book. 


\section{Allais on the Deduction}

Allais's account of the Transcendental Deduction plays an important structural role in the overall argument of her book. First, it removes one source of opposition to her account of the nature of intuition. In Chapter 7 Allais argues that intuitions 'do not depend on concepts to play their role of presenting us with particulars' (p.148). This conflicts with any reading of the Deduction which takes the conclusion of its argument to show the opposite: that the use of certain concepts - namely, the pure concepts are the understanding - is required in order for us to be presented with perceptual particulars. By providing an alternative reading of the Deduction on which its conclusion does not entail that the use of the categories is required in order for us to be presented with perceptual particulars, Allais removes one source of opposition to her account of the nature of intuition.

Second, Allais's account of the Deduction provides illustrative support for her general account of Kant's argument for transcendental idealism. Allais's claim is that Kant's argument for transcendental idealism turns specifically on the nature of a priori intuition: in broad terms, Kant's argument is that the a priori intuition of space and time is required in order for us to cognize the synthetic and a priori claims of mathematics, and that the transcendental ideality of space and time is entailed by their status as a priori intuitions. Her account of the Deduction, then, shows how its argument doesn't presuppose or entail transcendental idealism but rather can be combined with the idealism established in the Aesthetic to extend the conclusions of the Deduction to have wider scope.

Allais acknowledges that her account of the Deduction is only an account of one part of the Deduction, that section being a notorious hodgepodge or, perhaps, in non-evaluative terms, a recognised miscellany - of arguments and assertions. In particular, she does not attend to the role that self-consciousness or apperception play in the argument. Nevertheless, hers is an attempt to identify a clear and compelling line of reasoning which makes sense of Kant's claim in the Deduction that the application of the categories is necessary for our thought to have relation to an object. In this section and the next I set out Allais's reading of the Deduction. I will be 
concerned only with the general structure of her reading. This will unfortunately involve ignoring many of the interesting points of detail, but it will allow me, in the final sections, to raise some questions about that general structure.

Kant claims to vindicate the categories by showing them to have 'relation to an object' (p.267). Allais takes this to require showing that the categories can be used in 'a kind of referential thought: thought that succeeds in connecting with an object' (p.269). Concepts alone cannot connect us to objects: that is why cognition requires intuition. The problem posed by the categories, then, is that their objects are not given in either empirical or a priori intuition. So there is a prima facie problem with seeing how they can have relation to an object. Allais takes this problem to be dissolved by Kant showing that 'using the categories is a condition of having any successful referential thought about objects' (p.271). This is supposed to vindicate our use of the categories.

Allais's reading provides us with a transcendental argument. We start with something that Kant takes as uncontested: that we can successfully apply empirical concepts to the objects that are given to us in empirical intuition. One might think - if one is a sceptical empiricist, say - that our ability to do this doesn't require us to apply a priori concepts to the objects that are given to us in empirical intuition. The Deduction aims to show that this is not a real possibility: successful empirical concept application requires a priori concept application. Call this claim (AC). ${ }^{1}$

Allais's reconstruction of Kant's argument for (AC) turns on the generality and spontaneity of thought. In very broad terms, she sees Kant's argument as holding that empirical concept application requires representing the

\footnotetext{
${ }^{1}$ The term 'successful empirical concept application' is ambiguous. On one reading, empirical concept application is an achievement at which one can succeed or fail. Successful empirical concept application, on this reading, does not entail that one's application of the concept is correct. On another reading, successful empirical concept application requires that one apply the concept correctly. Conditions on the former will be conditions on the latter but not vice versa. Allais talks sometimes of what is required for empirical cognition (pp.262-3) which might suggest the latter reading, but whilst her account of cognition involves the claim that cognizing an object entails that the object exists, it doesn't commit her to the claim that cognizing an object requires that the attributes one predicate of the object be true. I won't pursue this issue here, though it raises issues structurally comparable to those discussed below.
} 
objects to which we apply empirical concepts as unified in a certain way, namely with necessity and universality, and that the application of the a priori concepts is required for the representation of this sort of unity (pp.271-285). There is much to say about this interesting proposal, both in terms of the merits of the argument, taken on its own terms, and in terms of whether it accurately captures Kant's argument. But I want to focus here on the claim (AC) itself.

Where does Kant's idealism enter this argument? Allais takes the idealism established in the Aesthetic to extend the scope of (AC) to take in all spatio-temporal objects (p.299). Allais's exposition of (AC) has it that if $x$ is an object of empirical cognition, then we must apply the categories to $\mathrm{x}$. Kant's idealism, on Allais's reading, involves a commitment to the claim that all spatio-temporal objects are possible objects of empirical cognition. Call this claim (TI). When combined with (AC), (TI) is said to entail that we must apply the categories to all spatio-temporal objects. ${ }^{2}$ This completes Kant's argument in the Deduction.

\section{Necessary Conditions}

Transcendental arguments are familiar to us but we need be careful about the formulation of their conditionals. When formulating Kant's argument in the Deduction we are wont to speak, as Allais sometimes does (p.260, p.261, p.298), of certain concepts being conditions on the possibility of something else. In the case of (AC), an instance of this practice would be the claim that that the pure concepts are conditions on the possibility of successful empirical concept application. But claims such as this are enthymematic: concepts themselves can't be conditions on the possibility of empirical cognition - their application, or their instantiation, or their possession may be, but concepts themselves aren't the right sort of things to feature as conditions. ${ }^{3}$ So we need to be clear about what exactly about the pure concepts is a condition on successful empirical concept application.

\footnotetext{
${ }^{2}$ Given that (TI) holds that all spatio-temporal objects are possible objects of empirical cognition, we're actually entitled only to a slightly weaker claim: namely, that it is possible for us to apply the categories to all spatio-temporal objects. I'll ignore this complication. ${ }^{3}$ It's an interesting question why this is so: perhaps there are restrictions on the terms which can occupy the $\mathrm{X}$-position in sentences of the form ' $\mathrm{X}$ is a condition of $\mathrm{Y}$ '.
} 
Allais's claim is 'the application of a priori concepts is a necessary condition of empirical concept application' (p.298). This clarifies the condition involved in (AC). But note that the application of a concept is different from its instantiation, and from the fact, if such it be, that the application of a priori concepts is a condition on empirical concept application, we can't conclude that the instantiation of a priori concepts is a condition on empirical concept application. Allais writes, in the full sentence from which the above quote is taken:

... that the application of a priori concepts is a necessary condition of empirical concept application is something that could be accepted by a realist; the argument (if successful) establishes the conditional claim that, if we can succeed in applying empirical concepts to an object, the categories apply to it. (p.298)

But this seems wrong, at least if the phrase 'the categories apply to [an object]' implicates that the categories correctly apply to the object. Concept application is compatible with concept non-instantiation as cases of mistaken identity show.

An example to illustrate the point: say that it is a condition on getting a date that one think of oneself as attractive. Imran gets dates. It follows that he must think of himself as attractive. Sadly for him, it doesn't follow that he is attractive. The requirement that one apply a concept in order that something be done is compatible with that concept failing to apply.

The difference between the application and instantiation of the categories has been raised before as an issue for interpretations of the Transcendental Deduction. ${ }^{4}$ But it presumes that there is a distinction to be drawn between the application and instantiation of the categories. And one might think that the difference between application and instantiation disappears in the framework of Transcendental Idealism where objects must conform to our conditions for cognizing them. So does Allais's invocation of Transcendental Idealism remove the distinction between application and instantiation? As far as I can see, it does not. On Allais's reading, (TI) extends the domain of (AC) from the domain of objects which are possible

4 (Cassam, 1987; Gomes, 2010, 2014; Rorty, 1970; Stroud, 1968; Van Cleve, 1999) 
objects of empirical cognition to all spatio-temporal objects. It follows from the combination of (AC) and (TI) that we must apply a priori concepts to all spatio-temporal objects. But this, again, is compatible with those concepts not being instantiated.

I said above that I lacked space to discuss Allais's reconstruction of Kant's argument for (AC) but one point is relevant here. As I have just noted, Allais's statements of her view suggest that she takes it that it is only the application of the a priori concepts, and not their instantiation, which is a condition on empirical concept application. Equally, her reconstruction of Kant's argument for (AC) entitles him to no more than this. The argument for (AC) turns on the fact that we need to represent objects in ways involving necessity and unity if we are to apply empirical concepts to them, and this is a claim about how we must represent objects - which is to say, about the kinds of concepts we must apply to objects. It is not a claim about the kinds of properties objects must instantiate in order for empirical concept application to be possible.

Could Allais's argument be amended so as to reach the stronger conclusion? On the face of it there are difficulties in supporting the claim that empirical concept application requires that the objects to which we apply concepts instantiate the categories. Since we can apply empirical concepts to objects which arguably don't instantiate the categories (afterimages, flashes, and so on), the claim would need to be restricted in some form: either to the claim that the application of empirical concepts to actual objects requires that the objects to which we apply concepts instantiate the categories, or to the claim that the application of empirical concepts to objects requires only that some of the objects to which we apply concepts instantiate the categories. But, again on the face of it, it's difficult to see how Allais's considerations about the spontaneity and generality of thought could motivate conditions on the application of empirical concepts to some restricted set of objects that weren't also conditions on the application of empirical concepts per se. Perhaps the argument could be amended to reach this different conclusion, but as presented it seems to support only the claim about application. 


\section{Which Problem?}

My concern thus far has been only to set out Allais's account of the Deduction, and to distinguish two claims: first, that the application of the categories is a requirement on empirical cognition; second, that the instantiation of the categories is a requirement on empirical cognition. Call these claims (APP) and (INS) respectively. I have suggested that Allais's commitment to (AC) should be understood as a commitment to (APP). The next thing to consider is the role Allais takes (APP) to play in a vindication of the categories. Allais is explicit that a vindication of the categories requires a proof that we are justified in using the categories. So the question we can ask is whether (APP) justifies our use of the categories.

What is required to justify our use of the categories? Allais takes it that the categories are justified when we are shown that they can relate to objects, and she tells us that 'concepts will lack relation to objects unless they apply to objects which are given to us in empirical intuition' (p.270). This claim admits of two readings. First, that the pure concepts relate to objects when it is possible to apply them to the objects of empirical intuition; second, that the pure concepts relate to objects when it is possible to correctly apply them to the objects of empirical intuition. Corresponding to these two notions of justification are two distinct construals of the aim of the Deduction. On one construal, the aim of the Deduction is to show that it is possible to apply the categories to the objects given in empirical intuition. On the second, the aim of the Deduction is to show that it is possible to correctly apply the categories to the objects given in empirical intuition.

One way to distinguish these differing understandings of the aim of the Deduction is to distinguish two distinct problems which Kant might be addressing in the Deduction. We can call the first Lambert's Problem, in reference to Lambert's letter to Kant of October 13, 1770 (C 10:105). ${ }^{5}$ In this letter, commenting on Kant's Inaugural Dissertation, Lambert raises the question of how it is possible for certain concepts to apply to the

\footnotetext{
${ }^{5}$ Kant texts are cited by the volume and page number in the Academy Edition of Immanuel Kant, Gesammelte Schriften (Berlin: Walter de Gruyter and predecessors, 1900-), with the exception of the Critique of Pure Reason which is cited in the standard A/B format.
} 
objects which are given to us in empirical intuition given the sharp distinction between sensibility and the understanding (10:105, 109). This problem is solved when it is shown possible to apply the pure concepts to the objects given in empirical intuition.

I will call the second, somewhat mischievously, Hume's Problem, with reference to one issue that Hume raises concerning our concept of cause. In his Enquiry Concerning Human Understanding, Hume asks 'how we arrive at the knowledge of cause and effect', suggesting that 'as a general proposition, which admits of no exception... the knowledge of this relation is not, in any instance, attained by reasonings a priori' (EHU 4.2.6-7). One problem this observation raises is that of accounting for the origin of our concept of cause, as Hume notes so perceptively in the Treatise of Human Nature (e.g., at 1.3.2). But it also raises a problem about our grounds for believing that there are any causes in the world, either as a general claim about events, or in the context of any particular causal judgement. This is the problem that comes to the fore in the Enquiry and Hume suggests that our grounds for believing in any particular instance of causation cannot be intuitive nor demonstrative (EHU 4.2.32). This suggests that we have no grounds for thinking any application of the concept of cause to be accurate and this problem can only be answered by showing that there are grounds for thinking that at least some of our applications of the concept of cause $d o$ apply the concept to objects which instantiate it.

Assume that the actuality of empirical cognition is not contested. Then Lambert's Problem is addressed when it is shown that the application of the categories is a requirement on empirical cognition. And Hume's Problem is addressed when it is shown that the instantiation of the categories is a requirement on empirical cognition. So, to return to our question, we can ask: which vindication is provided by (APP)? What kind of justification does it secure?

According to (APP), the application of the categories is a requirement on empirical cognition. But merely applying the categories is compatible with their not being instantiated. So (APP) can justify our use of the categories only if justification is understood as requiring nothing more than showing 
that it is possible to apply the categories to the objects of empirical intuition. Which is to say that (APP) vindicates our use of the categories only if a vindication is provided by solving Lambert's Problem. If justifying the categories requires showing that some of the categories are actually instantiated - if it requires a solution to Hume's Problem - then (APP) cannot help. Thus whether (APP) vindicates our use of the categories depends on what we think is required for justification and which problem we take ourselves to be addressing.

This prompts the question, of course, of which problem Kant took himself to be addressing. Or, since Kant himself says in the Prolegomena that the Critique of Pure Reason is the 'elaboration of the Humean problem in its greatest possible amplification' (Prol. 4:261; cf. 4:259-261), it prompts the question of whether Kant's Humean problem is what I have been calling 'Hume's Problem'. I'll return to this question in a moment. For now I just want to note that since Allais's account of the Deduction is committed to the mere application of the categories being conditions on empirical cognition, then it can only vindicate our use of the categories if justification of the categories doesn't require showing that the objects which are given in empirical intuition actually instantiate the categories. Allais's account of the Deduction answers Lambert's Problem, but it leaves open the possibility that the categories, like the concepts of fortune and fate, fail to correspond to any aspects of objects.

\section{Lambert or Hume?}

Let us take stock. Allais takes the Deduction to start with the actuality of successful empirical concept application. And she takes the main argument in the Deduction to claim that the possibility of applying empirical concepts to the objects given in intuition requires us to apply the pure concepts of the understanding to the objects given in empirical intuition. I have argued for two points. First, that this conditional is compatible with the objects given in empirical intuition failing to instantiate the pure concepts of the understanding. And second, that Allais's starting point and conditional will only vindicate our use of the categories if justification of the categories requires nothing more than showing that it is possible to apply them to objects. Any view which holds that vindication of the 
categories requires proving that at least some of our judgements involving the categories are accurate will not find vindication in Allais's reconstruction.

None of this tells against Allais's account of the Deduction, since it may be that Kant is only interested in showing that it is possible for us to apply the categories to the objects given in empirical intuition - that he is, in the terminology from my previous section, addressing only Lambert's Problem. In this final section I'll mention a couple of issues which bear on the question of whether this can be enough for Kant and connect them to one of the themes of Allais's book.

1. Putting to one side, for the moment, the question of whether Allais is right to ascribe this argument and its conclusion to Kant, what should we think of its merits? Without examining in detail the argument for (AC), any evaluation will be necessarily premature. But we might raise questions about the conclusion - about (AC) itself - independently of considering the argument in its support. According to (AC), the application of a priori concepts is a condition on the possibility of successfully applying empirical concepts. There are a number of ways one could cash out this claim but one natural way is to hold that someone who successfully applies an empirical concept to an object given in empirical intuition thereby thinks of it as falling under the categories.

Now consider the following judgement, made by a contemporary philosopher under the sway of Hume:

(1) That rock is not a substance.

According to (AC), someone who applies the empirical concept 'rock' to an object given in empirical intuition thereby thinks of it as falling under the categories, including that of substance. So someone who thinks of a rock as not a substance contradicts herself.

What should we make of this conclusion? In the heyday of Strawsonian conceptual analysis, such a characterisation would have been unremarkable. And in structure and outcome, Allais's account of the 
Deduction should remind us of some of Strawson's claims about the inconsistency involved in asserting that there are no other minds, or that there are no external objects. ${ }^{6}$ Still, I think it's fair to say that Strawson's claims have not found universal favour and one might worry about the attribution of inconsistency to such a judgement. (AC) seems to imply that either (1) is inconsistent or that the asserter of (1) is not making the same judgement that we would make were we to assert that sequence of English words. Someone impressed by Timothy Williamson's denial of conceptual truth will worry about either option (2007, ch.4). In particular, we might wonder what kind of mistake (semantic? grammatical?) the asserter of (1) is making and about the plausibility of any charge of linguistic incompetence.

This doesn't show that (AC) is false of course, and if there are ways in which we can apply concepts which do not involve using concepts in a judgement, then perhaps empirical concept application can require the application of a priori concepts without requiring that subjects think of objects as falling under the a priori concepts. Still, consideration of judgements of the form in (1) highlight the importance of getting clear on the kind of concept application at stake and may give us pause when considering the conclusion of Allais's reconstructed argument.

2. Consider now the question of whether the conclusion of Allais's reconstruction poses a challenge to Hume. Allais takes the argument to apply to the sceptical empiricist who thinks we can successfully apply empirical concepts to objects without applying a priori concepts to them (p.168, p.271, p.274) - and presumably Hume is one such sceptical empiricist. Now one reason Hume has for thinking that this state of affairs is possible is because he thinks there can be no a priori concepts. If this is the case, then the application of a priori concepts can't be a condition on empirical cognition. But in order to counter this line of reasoning, Hume would need to be convinced that there are such things as a priori concepts, and I don't think Allais takes Kant to be addressing concerns about the origin of a priori concepts in the Deduction in a way which would counter

\footnotetext{
${ }^{6}$ I have in mind Strawson's tendency to see such claims as, in a sense, tacitly inconsistent since their assertion contradicts claims which are conditions on the possibility of asserting such claims.
} 
the considerations Hume considers in the Treatise (THU 1.3.2) - even if the conclusion of the Deduction entails that there are such things. (Consider the framing of the Deduction in the first paragraph of $\$ 13$.) So I don't think this aspect of Hume's discussion can be that which is driving Allais's sceptical empiricist opponent.

Instead, Allais's thought seems to be that the sceptical empiricist denies that we need to represent necessary and universal connections in order to apply empirical concepts - a claim which Kant shows to be false in the Deduction. But, absent the concerns about our possession of a priori concepts, why should a Humean object to the claim that we need to represent necessary and universal connections in order to apply empirical concepts if that doesn't entail that the representation of necessary and universal connections is accurate? Consider that strand in Hume's thinking which accords Custom and Habit a special role in supporting chains of reasoning which cannot be supported by intuition or demonstration (EHU 5.1). It would be open, I think, for a contemporary Humean to accept Allais's reconstruction of Kant's argument, but hold that this shows that the way in which we are compelled to think about the world necessarily requires us to make use of notions of necessity and universality. That would show that Hume was wrong to think that we lack such concepts - but not that he was wrong to challenge our grounds for thinking that anything in the world corresponds to those notions. On this way of thinking about Allais's reconstruction, it is open for the sceptical empiricist to co-opt her argument as the continuation of their project by other means.

3. This bears on Kant's target in the Deduction. Would Kant have been satisfied by a conclusion which was compatible with the categories not being instantiated by any of the objects given in empirical intuition? Allais might agree that he would not - but counter that the argument against this possibility appears not in the Deduction but in the Analytic of Principles. Against that, we can consider $\$ 27$ of the Transcendental Deduction where Kant objects to preformation systems of pure reason on which 
the categories were neither self-thought a priori first principles of our cognition nor drawn from experience, but were rather subjective predispositions for thinking, implanted in us along with our existence by our author in such a way that their use would agree exactly with the laws of nature along which experience runs (B167)

He complains that

in such a case the categories would lack the necessity that is essential to their concept... I would not be able to say that the effect is combined with the cause in the object (i.e., necessarily), but only that I am so constituted that I cannot think of this representation otherwise than as so connected; which is precisely what the skeptic wishes most (B167-

168)

Preformation systems of pure reason establish only mere 'subjective necessity, arbitrarily implanted in us' (B168), a kind of necessity which falls short of what Kant wants to establish in the Deduction.

It seems plausible that one of Kant's targets in this passage is Hume. Kant says in the Metaphysical Foundations of Natural Science that preestablished harmony makes things 'only subjectively necessary, but objectively merely contingent, placing together, precisely as Hume has it when he calls this mere illusion from custom' (MFNS 4:476), and in the Prolegomena that Hume 'passes off... subjective necessity (i.e. habit) for an objective necessity (from insight)' (Prol. 4:257-8). And I take it that the charge being leveled, against Hume and others, is that they fail to ground the necessity of our application of categorial concepts in the objects themselves rather than in aspects of our own subjective dispositions.

This bears on the question of whether a successful Deduction requires the establishment of (APP) or (INS). For Kant's rejection of preformation systems of pure reason seems to involve the rejection of any view which establishes only the subjective necessity of the categories and not their objective necessity. And one way to put the worry about Allais's interpretation - indeed, of any interpretation of the Deduction that establishes only (APP) - is that it can do no more than this. Kant's 
comments in $\$ 27$ of the Deduction seem to suggest that this is not enough. $^{7}$

4. A final thought. Once we distinguish (APP) from (INS) we can see one source of motivation for the kind of conceptualism which Allais rejects. According to the conceptualist reading, intuitions depend on concepts to play their role in presenting us with particulars (p.148). One way to flesh out such a position would be to hold that we are presented in intuition with empirical objects which are perceptually represented as falling under the categories - through the involvement, say, of the understanding in synthesising the manifold of intuition. Allais rejects this view, of course but when combined with her account of transcendental idealism, it secures the objective necessity of the categories. At least, that's what I'll try to make plausible now.

The central notion in Allais's interpretation of transcendental idealism is the notion of an essentially manifest quality. Essentially manifest qualities are 'relational, mind-dependent qualities of things which can be present in perceptual experience, and which do not present us with qualities things have as they are in themselves, independent of their perceptually appearing to us' (p.124). There's much to say about this notion and the role it plays in Allais's account of transcendental idealism, but one useful way to think about essentially manifest qualities is as properties which objects have in virtue of their being perceptually represented as having that property. Essentially manifest qualities are thus a type of response-dependent property.

What kind of response determines the existence of essentially manifest qualities? Allais's answer is 'presence in perceptual experience': objects are $\mathrm{F}$ just in case they are necessarily perceptually presented as being $\mathrm{F}$, modulo various caveats about normal conditions and so on. (This is how I read the conditional which spans pp.121-122.) The kind of conceptualism I just gestured towards has the result that the objects we are given in empirical intuition are necessarily presented as being subject to the categories. It follows, from Allais's account of transcendental idealism, that

${ }^{7}$ (Gomes, 2014; Land, 2015) develop this line of thought. 
the objects of empirical intuition instantiate the categories - since all that is required for an object to instantiate an essentially manifest quality is for objects to be necessarily perceptually presented as instantiating that property. So the combination of conceptualism with Allais's account of transcendental idealism secures the move from (APP) to (INS) - and thus the objective necessity of the categories.

A similar result obtains if one combines Allais's account of the Deduction with a phenomenalist reading of transcendental idealism, at least if such a reading holds that the properties possessed by empirical objects are grounded in or determined by the properties which they are represented as having in experience (however Kant understood that notion). If this is right, then one way to think of phenomenalist and conceptualist views is as ways of bridging the gap between (APP) and (INS) and showing how the Deduction proves the instantiation of the categories.

To conclude, Allais's account of the Deduction can establish only the claim that the successful empirical concept application requires the application of the pure concepts of the understanding. This is compatible with the possibility that the objects given in empirical intuition fail to instantiate the pure concepts of the understanding. And if the success of the Deduction requires ruling out this possibility, then Allais's reconstruction, as interesting and ingenious as it is, leaves us silent in the face of Hume's arraignment. ${ }^{8}$

\footnotetext{
8 Thanks to Andrew Stephenson for helpful comments.
} 


\section{References}

Allais, L. (2015). Manifest Reality: Kant's Idealism and his Realism. Oxford: Oxford University Press.

Cassam, Q. (1987). Transcendental arguments, transcendental synthesis and transcendental idealism. Philosophical Quarterly, 37(149), 355378.

Gomes, A. (2010). Is Kant's Transcendental Deduction of the Categories Fit for Purpose? Kantian Review, 15(02), 118-137.

Gomes, A. (2014). Kant on Perception: Näive Realism, NonConceptualism, and the B-Deduction. The Philosophical Quarterly, 64(254), 1-19.

Hume, D. (1975). Enquiries concerning Human Understanding and concerning the Principles of Morals, L. A. Selby-Bigge and P.H. Nidditch (eds.), $3^{\text {rd }}$ ed. Oxford: Oxford University Press.

Hume, D. (1978). A Treatise of Human Nature, L.A. Selby-Bigge and P.H. Nidditch (eds.), 2nd ed, Oxford: Oxford University Press.

Kant, I. (1998). Critique of Pure Reason, P. Guyer and A.W. Wood (eds./trans.), Cambridge: Cambridge University Press.

Kant, I. (1999). Correspondence, A. Zweig (ed./trans.), Cambridge: Cambridge University Press.

Kant, I. (2002a). Prolegomena to Any Future Metaphysics That Will be Able to Come Forward as Science. In Theoretical Philosophy After 1781, H. Allison and P. Heath (eds.), G. Hatfield, M. Friedman, H. Allison, and P. Heath (trans.), Cambridge: Cambridge University Press.

Kant, I. (2002b). Metaphysical Foundations of Natural Science. In Theoretical Philosophy After 1781, H. Allison and P. Heath (eds.), G. Hatfield, M. Friedman, H. Allison, and P. Heath (trans.), Cambridge: Cambridge University Press.

Land, T. (2015). Nonconceptualist Readings of Kant and the Transcendental Deduction. Kantian Review, 20(1), 25-51.

Rorty, R. (1970). Strawson's Objectivity Argument. Review of Metaphysics, 24, 207-244.

Stroud, B. (1968). Transcendental Arguments. Journal of Philosophy, 65(9), 241-256.

Van Cleve, J. (1999). Problems from Kant. Oxford: Oxford University Press.

Williamson, T. (2007). The Philosophy of Philosophy. Oxford: Blackwell 